\title{
Kappa-opioid receptor activation during reperfusion limits myocardial infarction via ERK1/2 activation in isolated rat hearts
}

\author{
June Hong Kim ${ }^{1}$, Young Ho Jang ${ }^{1,2}$, Kook Jin Chun ${ }^{1}$, Jun Kim ${ }^{1}$, Yong Hyun Park ${ }^{1}$, Jeong Su Kim ${ }^{1}$, \\ Jin Mo Kim ${ }^{3}$, and Mi Young Lee ${ }^{3}$ \\ ${ }^{1}$ Institute of Cardiovascular Research Center, Pusan National University Yangsan Hospital, Yangsan, ${ }^{2}$ Department of Anesthesiology, \\ Pureun Hospital, ${ }^{3}$ Institute for Medical Science, Keimyung University, Daegu, Korea
}

Background: We investigated whether p42/p44 extracellular signal-regulated kinases (ERK1/2) and/or phosphatidylinositol-3-OH kinase (PI3K)-Akt play a crucial role in cardioprotection by $\kappa$-opioid receptor (KOP) activation.

Methods: Langendorff perfused rat hearts were subjected to $30 \mathrm{~min}$ of regional ischemia and $2 \mathrm{~h}$ of reperfusion. Antagonists of ERK1/2 and PI3K were perfused in hearts treated with the KOP agonist U50488H (U50). Infarct size was measured after $2 \mathrm{~h}$ of reperfusion. The phosphorylation states of ERK1/2 and Akt by Western immunoblots were determined. Drugs were perfused for a period of $5 \mathrm{~min}$ before and $30 \mathrm{~min}$ after reperfusion.

Results: Inhibition of ERK1/2 (26.8 $\pm 2.9 \%, \mathrm{P}<0.05$ vs. U50) but not PI3K (15.5 $\pm 1.1 \%, \mathrm{P}>0.05$ vs. U50) completely abrogated the anti-infarct effect of U50488H. Western blot analysis revealed a significant increase in ERK1/2 but not Akt phsophorylation in U50488H-treated hearts as compared to control hearts when measured immediately after reperfusion.

Conclusions: KOP activation effectively reduces myocardial infarction. The anti-infarct effect of U50488H is mediated by the ERK1/2, but not the PI3K-Akt pathway. (Korean J Anesthesiol 2011; 60: 351-356)

Key Words: Coronary occlusion, Heart, Myocardial function, Opioid receptors, Reperfusion.

\footnotetext{
Received: November 12, 2010. Revised: 1st, December 1, 2010; 2nd, December 9, 2010. Accepted: December 9, 2010.

Corresponding author: Young Ho Jang, M.D., Ph.D., Institute of Cardiovascular Research Center, Pusan National University Yangsan Hospital, Yangsan 626-770, Korea. Tel: 82-55-360-1457, Fax: 82-55-360-2204, E-mail: weonjo@pnuyh.co.kr

(ㄷ) This is an open-access article distributed under the terms of the Creative Commons Attribution Non-Commercial License (http:// creativecommons.org/licenses/by-nc/3.0/), which permits unrestricted non-commercial use, distribution, and reproduction in any medium, provided the original work is properly cited.
} 


\section{Introduction}

Opioids, which have been widely used clinically as anesthetic adjuvants or for pain control, can protect against myocardial ischemic-reperfusion (I/R) injury $[1,2]$. It has been well known that the activation of opioid receptors by ischemic preconditioning (I-Pre) or opioid-induced pharmacological pretreatment provides cardioprotection against myocardial I/R injury $[3,4]$. However, because pretreatment is seldom possible in acute myocardial infarction, ischemic postconditioning (I-Post) or reperfusion-targeted pharmacological strategies recently has generated considerable interest. Surprisingly, only a few studies thus far have investigated the effect of opioid receptor agonists administered solely during reperfusion in myocardial I/R injury. In addition, these studies were mainly focused on non-specific opioid receptor agonists or a $\delta$-opioid receptor (DOP) agonist $[2,5]$.

The $\mu-, \delta$-, and $\kappa$-opioid receptors have been characterized pharmacologically. Binding and developmental studies have identified the presence of DOP and $\kappa$-opiod receptors (KOP) in the adult rat heart [6]. KOP are localized in both adult and neonatal rat hearts [7]. Although there is controversy regarding the role of KOP in myocardial I/R injury, a recent report demonstrated that activation of KOP by U50488H administered as a single bolus prior to reperfusion provided cardioprotection in intact rat hearts [8]. In an our recent report, we also showed that KOP activation early in the reperfusion period reduced myocardial infarction and improved cardiac function in isolated rat hearts [9]. However, the exact intracellular mechanism of cardioprotection by KOP activation is not well known.

Interestingly, the activation of reperfusion injury salvage kinase (RISK) signaling pathways, such as the p42/p44 extracellular signal-regulated kinases (ERK1/2) and prosurvival phosphatidylinositol-3-OH kinase (PI3K)-Akt cascades, at the time of reperfusion exerts cardioprotection against lethal myocardial reperfusion injury [9]. Cardioprotection by nonspecific opioid receptor agonists and a selective DOP agonist is known to occur via the PI3K-Akt signaling pathway [2], while the involvement of the ERK1/2 pathway is unclear.

The purpose of this study was to assess the role of the RISK signaling pathway, ERK1/2 and PI3K-Akt, in cardioprotection by exogenous KOP activation during reperfusion.

\section{Materials and Methods}

The experimental procedures and protocols used in this study were reviewed and approved by our institutional animal care and use committee.

\section{Drugs and chemicals}

U50488H (a standard selective KOP agonist), LY294002 (a potent and selective PI3K inhibitor), and PD98059 (an ERK1/2 specific inhibitor) were purchased from Tocris Bioscience (Ellisville, MO). 2,3,5-Triphenyltetrazolium chloride (TTC) was obtained from Sigma-Aldrich Chemical (St. Louis, MO). Fluorescent polymer microspheres were purchased from Duke Scientific (Palo Alto, CA). Antibodies against Akt and ERK1/2 were purchased from Cell Signaling Technology Inc. (Beverly, MA). Other chemicals were obtained from Sigma-Aldrich Chemical.

\section{Langendorff isolated heart perfusion preparation}

Male Sprague-Dawley rats (Korea Taconic Co, South Korea), weighing 280-330 gm were used for the experiments. They received $100 \mathrm{mg} / \mathrm{kg}$ of pentobarbital sodium and $300 \mathrm{IU}$ of heparin intraperitoneally. Hearts were isolated and perfused with modified Krebs-Henseleit $(\mathrm{KH})$ solution containing (in $\mathrm{mM}) 118.5 \mathrm{NaCl}, 4.7 \mathrm{KCl}, 1.2 \mathrm{MgSO}_{4}, 1.8 \mathrm{CaCl}_{2}, 24.8 \mathrm{NaHCO}_{3}$, $1.2 \mathrm{KH}_{2} \mathrm{PO}_{4}$, and 10 glucose. Regional ischemia and reperfusion were induced as previously described [10]. In brief, a snare was made at the level of the proximal length of left coronary artery. Regional ischemia was induced by pulling the snare and confirmed by regional cyanosis and a substantial decrease in left ventricular developed pressure. Reperfusion was started by releasing the snare.

\section{Experimental protocol}

All hearts were subjected to $30 \mathrm{~min}$ of regional ischemia and 120 min of reperfusion, and each experimental group consisted of at least 7 hearts. In our previous report, the infarct volume as a percentage of ischemic volume in control hearts (CON) and $1 \mu \mathrm{M}$ U50488H treated hearts (U50) were $27.2 \pm 1.2 \%$ and $12.2 \pm 1.9 \%$, respectively $(\mathrm{P}<0.05)[9]$ and these results were compared to the following treatment groups to determine whether the RISK signaling pathway would play a critical role in KOP agonist-induced cardioprotection ( $\mathrm{n}=7$ for all groups): 1) U50 + LY; U50488H with $15 \mu \mathrm{M}$ of LY294002, 2) U50 + PD; U50488H with $20 \mu \mathrm{M}$ of PD98059, 3) LY; LY294002 alone, and 4) PD; PD98059 alone.

U50488H was dissolved in distilled water. LY294002 and PD98059 were dissolved in dimethyl sulfoxide. Drugs were perfused for a period of $5 \mathrm{~min}$ before and $30 \mathrm{~min}$ after reperfusion. The concentrations of all chemicals were based on our and other studies on isolated working rat hearts [9-11]. 
Table 1. Morphometrics for Isolated Rat Hearts

\begin{tabular}{lccccc}
\hline \multicolumn{1}{c}{ Group } & Body weight $(\mathrm{g})$ & Heart weight $(\mathrm{g})$ & LV volume $\left(\mathrm{cm}^{3}\right)$ & AR volume $\left(\mathrm{cm}^{3}\right)$ & AR/LV $(\%)$ \\
\hline CON & $305.0 \pm 5.8$ & $1.42 \pm 0.02$ & $0.519 \pm 0.020$ & $0.303 \pm 0.014$ & $58.7 \pm 2.6$ \\
U50 & $301.9 \pm 4.9$ & $1.40 \pm 0.01$ & $0.534 \pm 0.032$ & $0.307 \pm 0.021$ & $59.2 \pm 5.7$ \\
U50 + LY & $297.1 \pm 4.2$ & $1.31 \pm 0.03$ & $0.494 \pm 0.022$ & $0.302 \pm 0.009$ & $61.5 \pm 1.8$ \\
U50 + PD & $302.1 \pm 6.9$ & $1.42 \pm 0.03$ & $0.517 \pm 0.015$ & $0.351 \pm 0.012$ & $58.5 \pm 3.0$ \\
LY & $307.1 \pm 2.4$ & $1.37 \pm 0.05$ & $0.539 \pm 0.013$ & $0.302 \pm 0.016$ & $65.3 \pm 2.2$ \\
PD & $308.6 \pm 2.6$ & $1.39 \pm 0.05$ & $0.552 \pm 0.015$ & $0.376 \pm 0.023$ & $68.0 \pm 3.4$ \\
\hline
\end{tabular}

All values are mean \pm SEM. CON: control, LV: left ventricle, AR: area at risk, U50: $1 \mu \mathrm{M}$ of U50488H, LY: $15 \mu \mathrm{M}$ of LY294002, PD: $20 \mu \mathrm{M}$ of PD98059.

\section{Exclusion criteria}

We decided prospectively that any hearts with a heart rate $<$ 250 beats/min, or coronary flow $>18 \mathrm{ml} / \mathrm{min}$ or $<8 \mathrm{ml} / \mathrm{min}$ at the end of stabilization would be excluded from the study. Hearts failing to develop left ventricular systolic pressure $>80$ $\mathrm{mmHg}$ when the left ventricular end diastolic pressure was kept at 5-10 mmHg were also excluded. Any heart exhibiting arrhythmia during the stabilization period also was discarded.

\section{Determination of area at risk and infarct size}

At the end of each experiment (after $2 \mathrm{~h}$ of reperfusion), the area at risk (AR) and area of necrosis (AN) were measured by TTC stain and quantified with Image Tool (UTHSCSA Image Tool, version 3.0) as described previously [12]. In brief, the coronary artery was re-occluded and fluorescent polymer microspheres were infused. The hearts were weighed, frozen, and cut into 2-mm slices. The slices were incubated in $1 \%$ TTC in sodium phosphate buffer $37^{\circ} \mathrm{C}$ for $20 \mathrm{~min}$. The slices were immersed in $10 \%$ formalin and then identified by illuminating the slices with UV light. The areas were converted into volumes by multiplying area by slice thickness. The AN volume was expressed as a percentage of the AR volume. All measurements were performed in a blinded fashion. There were no significant differences in body weight, heart weight, LV volume, AR volume, and AR/LV among the groups (Table 1).

\section{Tissue lysate preparation and Western immunoblot analysis}

Additional hearts were randomly assigned into a control group $(n=6)$ and a $\mathrm{U} 50488 \mathrm{H}$ treatment group $(\mathrm{n}=6)$, and were subjected to $30 \mathrm{~min}$ of regional ischemia followed by $5 \mathrm{~min}$ of reperfusion. Myocardial samples were taken from the AR region at $10 \mathrm{~min}$ before and $5 \mathrm{~min}$ after reperfusion. Samples were homogenized in ice-cold lysis buffer. Equal amounts of protein $(50 \mu \mathrm{g} /$ lane $)$ were loaded, subjected to electrophoresis

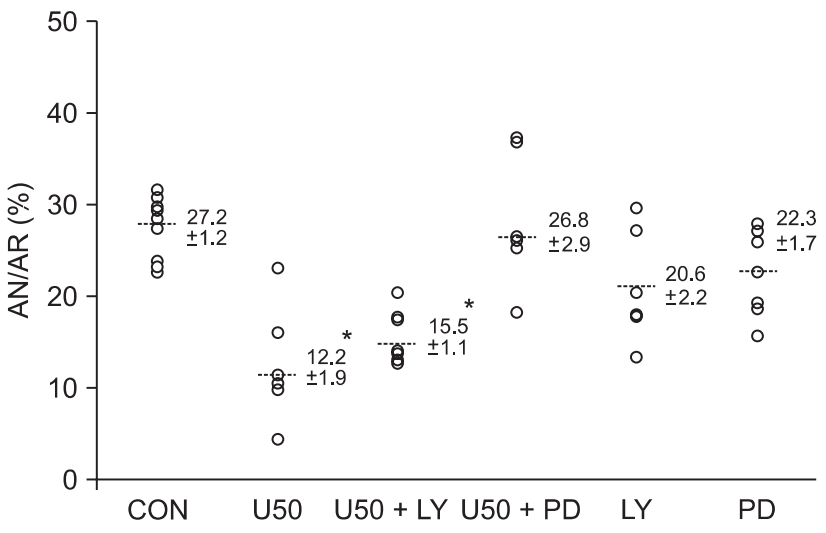

Fig. 1. Area at necrosis (AN) as a percentage of area at risk (AR), evaluated by triphenyltetrazolium chloride staining following 30 min of occlusion and $2 \mathrm{~h}$ reperfusion in an isolated rat heart model. Hearts were subjected to either control (CON), $1 \mu \mathrm{M}$ U50488H (U50), $15 \mu \mathrm{M}$ LY294002 (LY), $20 \mu \mathrm{M}$ PD98059 (PD), or U50 with LY or PD treatment. Each circle represents 1 heart. Horizontal bars depict mean of the group. AN: area of necrosis, AR: area at risk. Values are means \pm SEM. $* \mathrm{P}<0.05$ vs. CON.

on SDS-polyacrylamide gel and transferred to a polyvinylidene difluoride membrane. Membranes were blocked with nonfat milk and then incubated with primary antibodies that recognize phosphorylation of Akt (protein kinase B) and ERK1/2 at $4^{\circ} \mathrm{C}$ overnight. Bound primary antibodies were detected with a secondary anti-rabbit antibody and visualized by an enhanced chemiluminescence method. Data for total- and phosphoERK1/2 represent the sum of the 42- and 44-kDa bands.

\section{Statistical analysis}

Data are presented as means \pm SEM. Data analysis was performed with a personal computer statistical software package (SPSS for windows, Release 12.0; SPSS Inc, Chicago, IL). Data were analyzed using t-test and one-way analysis of variance (ANOVA) followed by a Bonferroni post-hoc testing. Differences were considered to be statistically significant when $P$ values were less than 0.05 . 


\section{Results}

\section{Infarct size studies with ERK1/2 and PI3K and inhibitors}

As shown in Fig. 1, the ERK1/2 inhibitor PD98059 (26.8 $\pm 2.9 \%)$ completely blocked the anti-infarct effect of U50488H $(\mathrm{P}<0.05$ vs. U50). However, there was no significant difference in AR/AN between the U50488H treatment group and the PI3K inhibitor LY294002 treatment group $(15.5 \pm 1.1 \%, \mathrm{P}>0.05$ vs. U50). LY294002 or PD98059 itself did not alter infarct size $(20.6 \pm 2.2 \%$ for $\mathrm{LY}$ and $22.3 \pm 1.7 \%$ for $\mathrm{PD}, \mathrm{P}>0.05$ vs. $\mathrm{CON})$.

\section{Western immunoblotting analysis of ERK1/2 and Akt}

To determine the involvement of the RISK pathway in the cardioprotective effect of KOP activation, we further measured ERK1/2 $\left(\mathrm{Thr}^{202} / \mathrm{Tyr}^{204}\right)$ and protein kinase B or Akt $\left(\operatorname{Ser}^{473}\right)$ phosphorylation during reperfusion in perfused rat hearts. ERK1/2 phosphorylation significantly increased after $5 \mathrm{~min}$ of reperfusion compared to $10 \mathrm{~min}$ before reperfusion in both groups (189.3 $\pm 95.0 \%$ in CON and 534.1 $\pm 283.1 \%$ in U50, Fig. 2). Furthermore, U50488H-treated hearts had significant increases
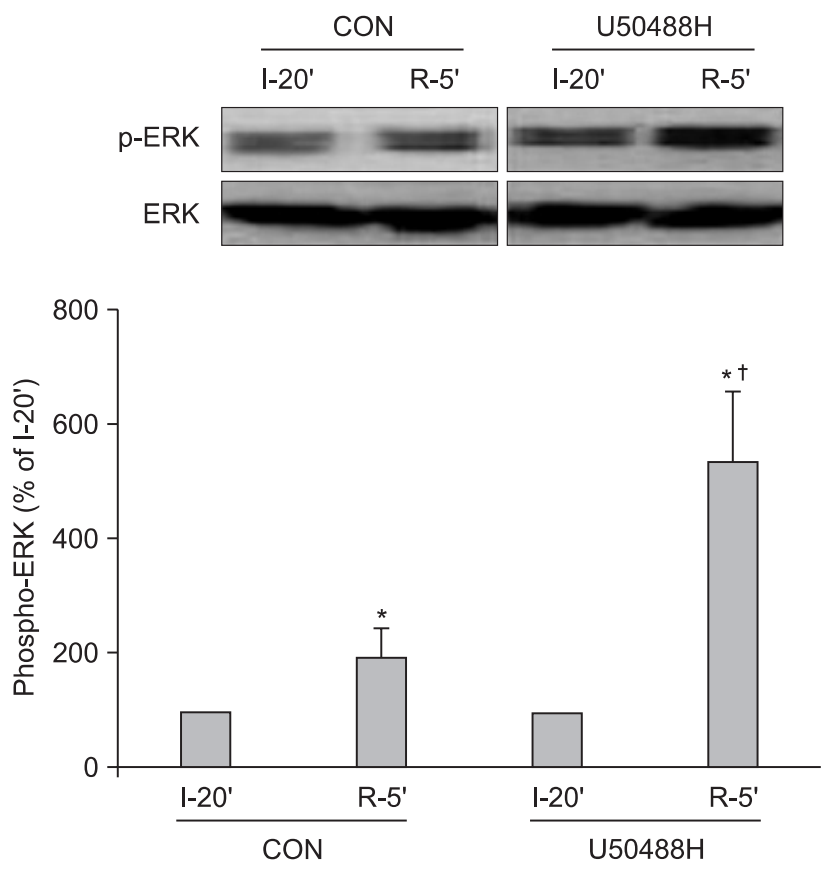

Fig. 2. Western immunoblotting analysis of phospho- and totalERK1/2 $\left(\mathrm{Thr}^{202} / \mathrm{Tyr}^{204}\right)$ in isolated rat hearts. Myocardial samples were obtained from the risk zone at $20 \mathrm{~min}$ of ischemia (I-20') and $5 \mathrm{~min}$ (R-5') after onset of reperfusion. Bar for R-5' is phosphorylation of proteins at $5 \mathrm{~min}$ of reperfusion relative to the value of I-20', expressed as means \pm SEM of individual experimental observations. CON indicates control hearts. ${ }^{*} \mathrm{P}<0.05$ vs I- $20^{\prime},{ }^{\dagger} \mathrm{P}<0.05$ vs. $\mathrm{R}-5$ ' (CON). in ERK1/2 phosphorylation compared to control hearts $(\mathrm{P}<$ $0.05)$ immediately after reperfusion. Myocardial sample taken from AR at 5 min of reperfusion revealed an increase in Akt phosphorylation compared with samples taken $10 \mathrm{~min}$ before reperfusion (303.1 $\pm 76.2 \%$ in CON and $242.9 \pm 46.2 \%$ in U50). However, there was no significant difference between the two groups $(\mathrm{P}<0.05$, Fig. 3$)$.

\section{Discussion}

In the present study, the anti-infarct effect by U50488H was totally abrogated by the ERK1/2 inhibitor PD98059. However, the PI3K inhibitor LY294002 could not block the infarct reducing effect of U50488H. And these results were corroborated by the phosphylation status of ERK1/2 and Akt kinases using Western immunoblott analysis.

The anti-infarct effect of U50488H in our previous study [9] was comparable with those previously reported for the nonspecific opioid receptor agonist morphine or the $\delta 1$-opioid receptor agonist BW373U86 [2]. And, these results are consistent with a recent report by the Gross group [8], who showed that administration of either DOP or KOP agonists as a single bolus
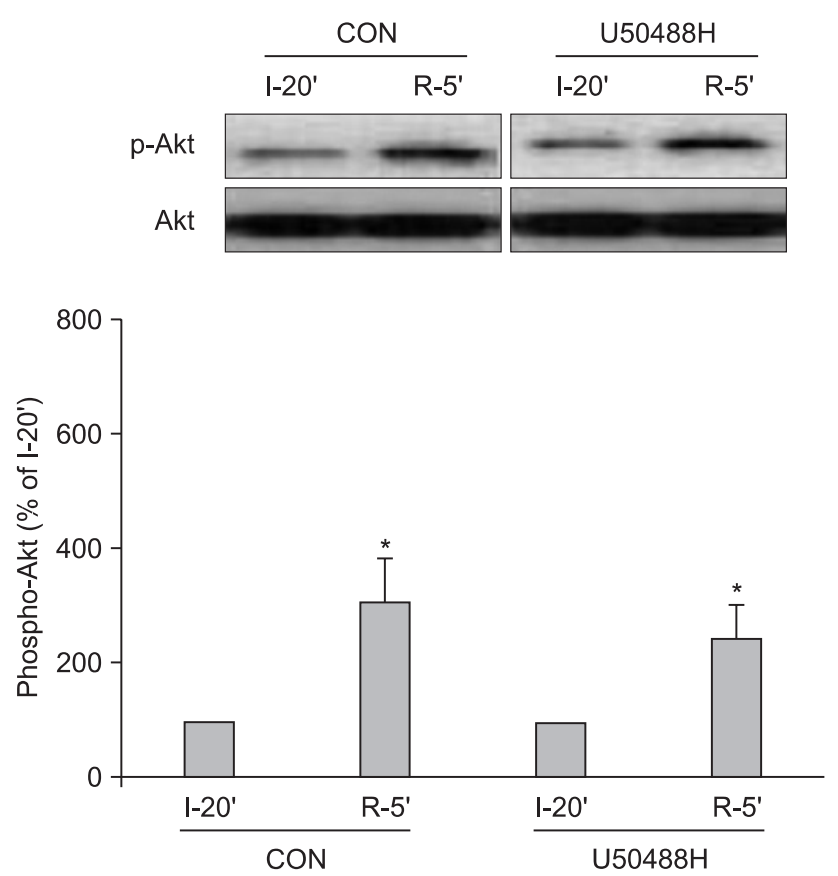

Fig. 3. Western immunoblotting analysis of phospho- and total-Akt $\left(\mathrm{Ser}^{473}\right)$ in isolated rat hearts. Myocardial samples were obtained from the risk zone at $20 \mathrm{~min}$ of ischemia (I-20') and $5 \mathrm{~min}$ (R-5') after onset of reperfusion. Bar for R- $5^{\prime}$ is phosphorylation of proteins at 5 min of reperfusion relative to the value of I-20', expressed as means \pm SEM of individual experimental observations. CON indicates control hearts. $* \mathrm{P}<0.05$ vs I- 20 . 
5 min prior to reperfusion provided infarct size-sparing effects in intact rat hearts. Collectively, these data strongly suggest that not only DOP but also KOP stimulation during the reperfusion phase plays a role in preventing lethal myocardial reperfusion injury.

The signaling mechanisms of opioid receptor activation are unveiling and seem to be similar to those of I-Pre and I-Post. In the present study, the infarct size-sparing effect of KOP activation by $\mathrm{U} 50488 \mathrm{H}$ was totally blocked by the ERK1/2 inhibitor PD98059 but not by the PI3K inhibitor LY294002, suggesting the involvement of ERK1/2 rather than PI3KAkt signaling pathway in cardioprotection by KOP activation during reperfusion. This finding was also corroborated by direct measurement of ERK1/2 and Akt kinases using Western blots. Meanwhile, Peart and colleagues [13] reported that pretreatment with the PI3K inhibitor wortmannin abolished infarct reduction by $\mathrm{U} 50488 \mathrm{H}$ in in vivo rat hearts. Of note, however, we measured infarct size using ERK1/2 and PI3K antagonists in KOP activation in conjunction with assessing the phosphorylation status. Our present study highly implicates the involvement of ERK1/2 but not PI3K-Akt in the cardioprotection achieved with KOP activation during reperfusion. We do not know the exact reason for the discrepancy of results between our and Peart's group, but the two pathways may also follow different spatio-temporal routes by KOP activation at early reperfusion, which should be defined in the future.

Meanwhile, the survival kinase cascades ERK1/2 and PI3KAkt may exhibit 'cross-talk' such that inhibiting of one cascade may activate the other and vice versa [14]. However, it does not seem that cross-talk always occurs. For instance, a recent study stressed the pivotal role of ERK1/2 but not PI3K/Akt in I-Post mediated cardioprotection [15]. In this regard, the relative importance of the two kinases in mediating the protection by KOP activation remains controversial. The results from our study suggest that ERK1/2 but not PI3K/Akt is the predominant mediator of KOP-induced protection.

Interestingly, some drugs protect the myocardium against ischemic but not against reperfusion injury and vice versa [16]. Accordingly, when administering drugs to prevent myocardial $\mathrm{I} / \mathrm{R}$ injury, it is important to know whether it is protective only when given before ischemia and/or when given after ischemia during reperfusion. In this regard, it has been well accepted that DOP stimulation is effective not only during the pre-ischemic period but also during the reperfusion period [17]. Although it seems that KOP is less influential in I-Post [18], there have been data to suggest that exogenous activation of KOP have an infarct-sparing effect in the myocardial I/R model. Peart and colleagues [19] reported that exogenously induced KOP activation by three different KOP agonists (U50488, ICI204448, and BRL52537) $10 \mathrm{~min}$ before the onset of ischemia reduced infarct size in an intact rat model of myocardial infarction. However, thus far there is little in scientific examining the effect of infarct-sparing associated with KOP activation by sustained drug administration during the reperfusion phase. Combined with previous reports of cardioprotection by KOP treatment before index ischemia [12,19], our result strongly suggest that KOP activation confers cardioprotection not only when given before ischemia but also when given after ischemia during the reperfusion phase.

In summary, our results suggest that sustained KOP activation targeting the reperfusion phase confers cardioprotection by the ERK1/2 subcellular signaling pathway but not the PI3K-Akt pathway. KOP activation during reperfusion, therefore, might be another cardioprotective mechanism.

\section{References}

1. Zaugg M, Lucchinetti E, Uecker M, Pasch T, Schaub MC. Anaesthetics and cardiac preconditioning. Part I. Signalling and cytoprotective mechanisms. Br J Anaesth 2003; 91: 551-65.

2. Gross ER, Hsu AK, Gross GJ. Opioid-induced cardioprotection occurs via glycogen synthase kinase beta inhibition during reperfusion in intact rat hearts. Circ Res 2004; 94: 960-6.

3. Okubo S, Tanabe Y, Takeda K, Kitayama M, Kanemitsu S, Kukreja $\mathrm{RC}$, et al. Ischemic preconditioning and morphine attenuate myocardial apoptosis and infarction after ischemia-reperfusion in rabbit: role of delta-opioid receptor. Am J Physiol Heart Circ Physiol 2004; 287: H1786-91.

4. Xia Q, Zhang WM, Shen YL, Wong TM. Decreased affinity for $\mathrm{K}$-receptor binding during reperfusion following ischaemic preconditioning in the rat heart. Life Sci 1996; 58: 1307-13.

5. Chang WL, Lee SS, Su MJ. Attenuation of post-ischemia reperfusion injury by thaliporphine and morphine in rat hearts. J Biomed Sci 2005; 12: 611-9.

6. Ventura C, Bastagli L, Bernardi P, Caldarera CM, Guarnieri C. Opioid receptors in rat cardiac sarcolemma: effect of phenylephrine and isoproterenol. Biochim Biophys Acta 1989; 987: 69-74.

7. Zimlichman R, Gefel D, Eliahou H, Matas Z, Rosen B, Gass S, et al. Expression of opioid receptors during heart ontogeny in normotensive and hypertensive rats. Circulation 1996; 93: 1020-5.

8. Gross ER, Gross GJ. Ligand triggers of classical preconditioning and postconditioning. Cardiovasc Res 2006; 70: 212-21.

9. Lee YC, Jang YH, Kim JM, Kim AR, Kim CJ, Kim YN. Effect of a kappa-opioid receptor agonist U50488H given at early reperfusion phase in isolated rat hearts. Korean J Anesthesiol 2008; 54: S29-34.

10. Hausenloy DJ, Tsang A, Yellon DM. The reperfusion injury salvage kinase pathway: a common target for both ischemic preconditioning and postconditioning. Trends Cardiovasc Med 2005; 15: 69-75.

11. Yu XC, Wang HX, Pei JM, Wong TM. Anti-arrhythmic effect of kappa-opioid receptor stimulation in the perfused rat heart: involvement of a cAMP-dependent pathway. J Mol Cell Cardiol 1999; 31: 1809-19.

12. Jang Y, Xi J, Wang H, Mueller RA, Norfleet EA, Xu Z. Postcon- 
ditioning prevents reperfusion injury by activating delta-opioid receptors. Anesthesiology 2008; 108: 243-50.

13. Peart JN, Gross ER, Reichelt ME, Hsu A, Headrick JP, Gross GJ. Activation of kappa-opioid receptors at reperfusion affords cardioprotection in both rat and mouse hearts. Basic Res Cardiol 2008; 103: 454-63.

14. Hausenloy DJ, Mocanu MM, Yellon DM. Cross-talk between the survival kinases during early reperfusion: its contribution to ischemic preconditioning. Cardiovasc Res 2004; 63: 305-12.

15. Darling CE, Jiang R, Maynard M, Whittaker P, Vinten-Johansen J, Przyklenk K. Postconditioning via stuttering reperfusion limits myocardial infarct size in rabbit hearts: role of ERK1/2. Am J Physiol Heart Circ Physiol 2005; 289: H1618-26.

16. Ebel D, Lipfert P, Frässdorf J, Preckel B, Müllenheim J, Thämer V, et al. Lidocaine reduces ischaemic but not reperfusion injury in isolated rat heart. Br J Anaesth 2001; 86: 846-52.

17. Förster K, Kuno A, Solenkova N, Felix SB, Krieg T. The delta-opioid receptor agonist DADLE at reperfusion protects the heart through activation of pro-survival kinases via EGF receptor transaction. Am J Physiol Heart Circ Physiol 2007; 293: H1604-8.

18. Zatta AJ, Kin H, Yoshishige D, Jiang R, Wang N, Reeves JG, et al. Evidence that cardioprotection by postconditioning involves preservation of myocardial opioid content and selective opioid receptor activation. Am J Physiol Heart Circ Physiol 2008; 294: H1444-51.

19. Peart JN, Gross ER, Gross GJ. Effect of exogenous kappa-opioid receptor activation in rat model of myocardial infarction. J Cardiovasc Pharmacol 2004; 43: 410-5. 